\title{
Effect of self-ion irradiation on the microstructural changes of alloy EK-181 in annealed and severely deformed conditions
}

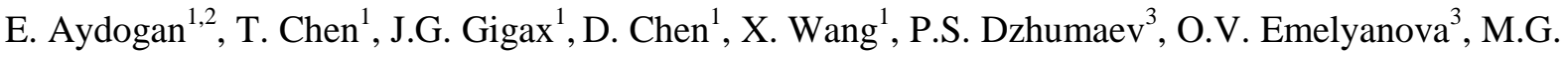
Ganchenkova $^{3}$, B.A. Kalin ${ }^{3}$, M. Leontiva-Smirnova ${ }^{3}$, R.Z. Valiev ${ }^{4,5}$, N.A. Enikeev ${ }^{4}$, M.M. Abramova $^{4}$, Y. Wu ${ }^{6}$, W.Y. Lo $^{6}$, Y. Yang ${ }^{6}$, M. Short ${ }^{7}$, S.A. Maloy ${ }^{2}$, F.A. Garner ${ }^{1,3}$, L. Shao, ${ }^{1, *}$

1. Texas A\&M University, College Station, TX 77840, USA

2. Los Alamos National Laboratory, Los Alamos, NM 87545, USA

3. National Research Nuclear University (MEPhI), Moscow, Russian Federation

4. Institute of Physics of Advanced Materials and Nanocenter, Ufa State Aviation Technical University, Ufa, Russian Federation

5. Saint Petersburg State University, St. Petersburg, Russian Federation

6. University of Florida, Gainesville, FL 32611, USA

7. Massachusetts Institute of Technology, Cambridge, MA 02139, USA

\begin{abstract}
EK-181 is a low-activation ferritic/martensitic steel that is an attractive candidate for in-core component materials for both fast reactors and fusion reactors. To assess the effect of structural engineering on radiation response, two variants of EK-181 were studied: one in an annealed condition and the other subject to severe plastic deformation. These specimens were irradiated with $3.5 \mathrm{MeV} \mathrm{Fe}$ self-ions up to 400 peak displacements per atom (dpa) at temperatures ranging from $400{ }^{\circ} \mathrm{C}$ to $500{ }^{\circ} \mathrm{C}$. The deformation did not suppress swelling over the whole irradiated region. Instead, deformed samples showed higher swelling in the near surface region. Void swelling was found to be correlated with grain boundary instability. Significant grain growth occurred when steady-state void growth started.
\end{abstract}

*Corresponding author at: 308 Bizzell East, Dept. of Nuclear Engineering, Texas A\&M University, College Station, TX 77843. Tel.: 979845 4107; fax: 979845 6443. Email address: 1shao@tamu.edu (L. Shao). 


\section{Introduction}

In-core structural materials for next-generation nuclear reactors require good radiation resistance to embrittlement and void swelling, but also require a rapid reduction of neutroninduced activity to meet low-activation goals [1-4]. Among various high-chromium reducedactivation ferritic/martensitic (F/M) alloys [1,5,6], the Russian alloy EK-181, often designated as "Rusfer", has been intensively studied as one promising candidate for in-core components for both fast reactors and fusion reactors [7]. Its popularity arises from the potentially lower swelling, low activation, and high strength, especially at higher irradiation temperatures $[7,8]$. The alloy is normally used in the quenched and tempered condition, yielding a dual phase tempered F/M structure [7,9]. Furthermore, extensive research efforts have been and continue to be made to improve this alloy and its variants through the optimization of heat treatments and surface modification to produce better corrosion resistance and mechanical properties [1,7,9-15].

Previous studies have shown that nano-grained metals often exhibit improved swelling resistance due to defect sink properties of high density of nano-grain boundaries [16]. Void swelling studies on nano-grained EK-181 have not yet been performed and are critical to evaluate the impact of this processing technique for further increasing its radiation tolerance. Severe plastic deformation (SPD) techniques which allow nano-structuring of a wide range of bulk metallic materials appear to be a promising tool for producing high strength and radiation-resistant materials.

Early studies on the ion irradiation of alloys treated by severe plastic deformation (SPD) suggest that they often develop significantly enhanced radiation tolerance [17-20]. Equal channel angular pressing (ECAP), one SPD technique described in Ref [21,22], has been used to introduce ultrafine grains in both austenitic and F/M alloys. Studies on austenitic Fe-14Cr16Ni and F/M T91 have shown that ECAP can improve swelling resistance due to increased defect sink densities arising from very high interface-to-volume ratios introduced by SPD [23-25]. In addition to increases in grain boundary densities, large shear stresses involved in the ECAP process result in fragmentation and redistribution of second phase particles $[26,27]$. Several studies have shown that ion irradiation results in compositional and size changes of nano-structured precipitates occurring together with grain boundary segregation $[18,28]$. The overall structural stability of SPD-introduced features depends strongly on material composition and fabrication. The first neutron irradiation experiments on ECAPed 
low carbon steel [29,30] and ECAPed 321 stainless austenitic steel [31] demonstrated the potential to produce more radiation-tolerant materials for fission reactor environments.

Since the EK-181 alloy has a substantial and growing database for both unirradiated and neutron-irradiated conditions, it is ideal to use high dose self-ion irradiation as a surrogate to neutron irradiation for accelerated screening of different processing techniques. Comparison of the microstructural evolution of ion irradiated specimens with neutron irradiation data can validate behaviors observed in ion irradiated specimens and further refines ion irradiation testing. The present study is part of a larger effort where EK-181 is being irradiated in an annealed ferritic condition, an F/M condition, and in several severely plastically deformed conditions. In this report we focus only on two conditions, namely annealed ferritic EK-181 and one severely deformed variant produced from the annealed condition.

\section{Experimental Procedure}

The composition of the EK-181 alloy used in this study is given in Table 1. In order to study the effect of nano-structuring on the irradiation response, EK-181 was produced in two variants. The first variant was hot rolled and annealed at $800{ }^{\circ} \mathrm{C}$ for 1 hour, slowly cooled in a furnace down to $600{ }^{\circ} \mathrm{C}$, and then air cooled. This variant is referred as the "annealed" condition in the following discussion. The annealed samples were received in the form of 10 mm diameter rods.

The second variant was produced starting from the annealed condition. The samples were subjected to high pressure torsion (HPT), a method of severe plastic deformation that introduces extremely high strain into processed materials [32]. This variant is referred as 'severely deformed' condition in the present study. The HPT technique is schematically shown in Fig. 1. HPT was performed with an applied pressure of $6 \mathrm{GPa}$ at $400{ }^{\circ} \mathrm{C}$, and at 0.5 rotations per minute. In this study, 10 rotations of the die-set were performed. As a result, nano-structured specimens of EK-181 steel were produced in the form of disks, $10 \mathrm{~mm}$ in diameter and $0.9 \mathrm{~mm}$ in thickness. The imposed shear strain at half-radius was estimated by $\gamma=2 \pi r n / h$ (where $r$ is the distance from a disk center, $h$ is the thickness of the disk and $n$ is the number of rotations), leading to $\gamma \approx 175$.

EK-181 samples of both variants were cut into thin disks of $\sim 1 \mathrm{~mm}$ thickness. The specimen thickness was further reduced down to about $600 \mu \mathrm{m}$ by applying mechanical polishing using 
$\mathrm{SiC}$ paper grit of 800 increasing to 4000 . The mechanically polished samples were then chemically polished in a solution of sulfuric acid (50\%) and phosphoric acid at $20{ }^{\circ} \mathrm{C}$ with an applied voltage of $5 \mathrm{~V}$ for 20-30 seconds to remove surface damage introduced by mechanical polishing.

Samples were irradiated by using $3.5 \mathrm{MeV} \mathrm{Fe}^{2+}$ ions to 100 peak dpa at 400, 425, 450, 475 and $500{ }^{\circ} \mathrm{C}$, to determine the temperature range of swelling under these ion irradiation conditions. In another set of experiments, samples were irradiated at the previously determined peak swelling temperature of $475^{\circ} \mathrm{C}$ to higher doses of 200,300 and 400 peak dpa. Fig. 2 shows the calculated dose and implanted Fe distributions for a peak dose of 100 dpa as a function of depth using the Monte Carlo simulation code SRIM [33]. Due to the recent finding that the full damage cascade option in SRIM overestimates the damage creation by a factor of two, calculations were obtained using the recommended KinchinPease option with displacement energy of $40 \mathrm{eV}$ for consistency with the neutron damage code SPECTER [34,35]. For 3.5 MeV Fe ions, the peak projected ion range (Rp) is $\sim 1.2 \mu \mathrm{m}$. The resultant dpa peak is located at a depth of $\sim 1.0 \mu \mathrm{m}$.

The vacuum maintained during ion irradiations was better than $1 \times 10^{-6}$ Torr. A $6 \mathrm{~mm} \times 6 \mathrm{~mm}$ defocused ion beam was used, rather than a rastering or beam-sweeping mode, due to the fact that rastering suppresses void swelling [36,37]. Swelling suppression by rastering has been experimentally demonstrated in two recent studies $[38,39]$. The dose uniformity within a 6 $\mathrm{mm} \times 6 \mathrm{~mm}$ irradiated region was confirmed by Fe ion irradiation on a monocrystalline $\mathrm{Si}$ sample, following by channeling Rutherford Backscattering Spectrometry analysis by using a $2 \mathrm{MeV} \mathrm{He}$ analysis beam with $1 \mathrm{~mm}$ beam spot size. The Fe ion-induced damage varied less than $5 \%$.

The beam current was controlled at $\sim 200 \mathrm{nA}$ to minimize localized beam heating, with the temperature rise estimated to be at $\leq 5^{\circ} \mathrm{C}$. The sample temperature was monitored by using a thermocouple mounted on the side of the specimen mounting stage. The thermocouple provided feedback to the power supply controlling the heating filament within the hot stage positioned beneath the specimen mounting holder. The temperature fluctuation recorded during the irradiation was less than $\pm 5^{\circ} \mathrm{C}$. The displacement rate at the peak displacement depth was calculated using the SRIM code to be $1.7 \times 10^{-3}$ dpa per second. 
The original microstructure was characterized by the MEPhI coauthors in Russia using a Zeiss EVO 50 XVP scanning electron microscope (SEM), followed by additional transmission electron microscopy at Texas A\&M University using an FEI Tecnai F20 ST transmission electron microscope (TEM) operating at $200 \mathrm{kV}$. Scanning transmission electron microscopy (STEM) and bright field (BF) imaging at an under-focus condition of $\sim 500 \mathrm{~nm}$ were used for the swelling studies. Grain size measurements were also performed by using BF imaging. The lamella specimens for cross-sectional TEM were prepared by using a focused-ion-beam (FIB) lift-out technique. Prior to FIB cutting, a thin Pt layer was deposited on the sample surface to protect the features beneath the surface. The FIB specimen thickness was determined by using electron energy loss spectroscopy (EELS) for each specimen. Typical lamella thicknesses ranged from $50 \mathrm{~nm}$ to $100 \mathrm{~nm}$.

The present study focuses on the swelling resistance and grain stability during irradiation of the two EK-181 variants. Therefore, a comparison was always made for the irradiated region $\leq \mathrm{Rp}$ and the region far beyond $\mathrm{Rp}$, in order to differentiate irradiation and thermal annealing effects on grain growth.

\section{Results and Discussion}

Fig. 3a and 3b compare the SEM surface imaging of annealed EK-181 and severely deformed EK-181, respectively, prior to ion irradiation. The grain sizes of the annealed sample ranged from $\sim 1$ to $10 \mu \mathrm{m}$. Furthermore, carbides, shown by arrows, were found to form along grain boundaries. For the severely deformed sample, grains were observed to be homogenous and equiaxed. This suggests that there was no large strain gradient across the deformed samples. Grain size measurements for the severely deformed samples is challenging for SEM analysis due to its resolution limit. TEM characterization, averaged over more than 100 grains, showed that the grain size is reduced to $\sim 135 \mathrm{~nm}$ after HPT deformation.

\subsection{Swelling}

\subsubsection{Temperature dependent swelling}

Fig. 4a and 4b show typical TEM micrographs obtained from the annealed and severely deformed samples, respectively, after irradiation to 100 peak dpa at 400, 425, 450, 475 and $500{ }^{\circ} \mathrm{C}$. With increasing temperature, the void size increased and the void density decreased. 
In all samples, voids formed only within a shallow region near the front portion of the ion range, less than $300 \mathrm{~nm}$ from the surface. No voids were observed at deeper regions, similar to the behavior observed in earlier studies in pure iron arising first from the spatial difference in interstitial and vacancy concentrations along the ion path, and second from the excess interstitial concentration deposited in the region close to the projected range of implanted $\mathrm{Fe}$ [40]. The combination of these two effects is generally referred to as "defect imbalance".

Comparison between the temperature dependence of swelling of the annealed and severely deformed samples shows that severely deformed samples exhibited higher swelling than annealed samples. Furthermore, maximum swelling occurred at $475{ }^{\circ} \mathrm{C}$ for both conditions, a temperature that is in agreement with results of earlier studies on similar alloys $[3,41,42]$. Due to this reason, our studies on swelling dependence on dpa selected $475^{\circ} \mathrm{C}$ as the irradiation temperature for higher dose specimens.

\subsubsection{Dose dependent swelling}

Fig. 5a-d show TEM micrographs of typical void distributions in annealed EK-181 samples irradiated at $475^{\circ} \mathrm{C}$ to $100,200,300$ and 400 peak dpa. Both void density and size increased with increasing dpa. For the sample at 100 peak dpa, the mean void size was determined to be $\sim 7 \mathrm{~nm}$, and increased to $\sim 85 \mathrm{~nm}$ in the 400 peak dpa sample. Fig. 5e shows the depthdependent swelling curves for the four dpa levels, superimposed on the SRIM-calculated dpa and injected Fe ion profiles. With increasing dpa, void swelling was pushed into deeper regions and the swelling profile began to resemble the dpa curve more closely. However, the injected interstitial effect strongly suppressed void swelling throughout much of the iondeposited range. This suppression is a well-known phenomenon where void nucleation is strongly suppressed by orders of magnitude, and the post-transient swelling rate is often suppressed by a factor of 3 to 5 [43].

Void characteristics measured over a depth range of 400 to $700 \mathrm{~nm}$ are shown in Fig. 6. This depth interval is ideal to minimize both the injected interstitial and surface sink effects. With increasing dpa, voids grew and began to approach saturation to a size of $\sim 70 \mathrm{~nm}$. Figs. $7 \mathrm{a}-\mathrm{d}$ show typical micrographs of void distributions in severely deformed EK-181 samples irradiated at $475{ }^{\circ} \mathrm{C}$ to $100,200,300$ and 400 peak dpa. Fig. 7e shows the depth dependent swelling curves for different dpa levels. Similar to the annealed samples, defect imbalance effects are still dominant even for the highest dpa and voids are suppressed in most of the 
injected ion range. Compared to the annealed specimens, however, void distributions in the deformed samples peak closer to the front surface with the maximum swelling in the region of $100 \mathrm{~nm}$ to $400 \mathrm{~nm}$ depth for all peak dpa levels. Figure 8a and 8b show the void size and density changes as a function of dpa at the depth range of $400 \mathrm{~nm}$ to $700 \mathrm{~nm}$. The void size increased with dpa and appeared to saturate at $\sim 21 \mathrm{~nm}$ while the void density remained almost constant. In contrast to the annealed samples, the observed voids are smaller but present at higher concentration in the deformed samples.

\subsubsection{Swelling comparison}

Swelling peaked at about half of the ion projected range for the annealed sample. But the maximum swelling depth shifted to a much shallower depth region for the deformed samples. At this shallow region (100 to $400 \mathrm{~nm}$ ), deformed samples swelled more than annealed samples. However, if the comparison is made at the half of the ion projected range, the annealed samples swelled more than the deformed samples. In order to address this issue, we compare the swelling as a function of local dpa over the sampled depth increment for the two sets of samples, with more focus on the swelling incubation period difference, rather than the absolute swelling values over the entire damaged range, yielding the result that the near surface regions of the deformed samples have the shortest swelling incubation periods.

Due to the combined influence of the gradients in dpa and injected interstitials, the resultant depth distribution of swelling can be challenging to analyze. It is not surprising that the results might be sensitive to the depth range chosen for void counting. When searching for void characteristics, we chose relatively wide depth intervals.

To demonstrate the sensitivity of void swelling to both local dpa and local depth, Fig. 9a and $9 \mathrm{~b}$ plot the swelling curves at different depth regions for annealed and severely deformed samples, respectively. For the annealed samples, the void swelling incubation periods varied at different depths. The deeper the depth, the longer the incubation period, primarily due to a strong injected interstitial as the peak dpa location is approached. A secondary contribution to increasing incubation periods was discussed in an early paper by Garner and Guthrie who assessed the possible impact of "internal gradients" in dpa rate on the resultant swelling vs. depth profile [44]. Of most importance, Garner et al. showed that higher dpa rates frequently extended the transient regime of swelling in neutron irradiations of austenitic steels $[45,46]$. It was also shown in the previous studies that ion-induced swelling of simple model austenitic 
alloys exhibited an incubation period that increased with increasing dpa rate [47]. Variation of incubation periods in the severely deformed samples appear to be much less since swelling occurs in a relatively narrower and shallower region where both the dpa rate effect and injected interstitial effect are weaker.

Since the maximum swelling in the deformed samples occurs at shallow depths which correspond to low local dpa region, the final swelling is not as significant as that in annealed samples. This leads to the difference that, while the annealed samples reach the anticipated steady-state swelling of $\sim 0.2 \% / \mathrm{dpa}$ at all depths sampled, such behavior is not observed in the severely deformed samples. Only the depth region of 100 to $400 \mathrm{~nm}$ of the deformed sample reaches the steady-state swelling rate of $\sim 0.2 \% / \mathrm{dpa}$, with an incubation period of $\sim 75$ local dpa. In a comparison with Fig. 9a, this incubation period is the lowest observed in all samples in this study, regardless of the analysis depth.

Since the shallow swelling regions are not affected by the injected interstitial effect, the swelling dependence on depth can be converted to swelling dependence on local dpa values, thus obtaining useful information on the rate of swelling per dpa. Fig. 10a plots swelling depth profile obtained from the annealed sample after 400 peak dpa irradiation. The solid circles denote the region unaffected by injected interstitials and the hollow circles denote the regions where injected interstitials begin to suppress swelling. Fig. 10b plots the swelling values as a function of local dpa values, extracted from Fig. 10a. We once again see a swelling rate in the injected-interstitial-free zone that is comparable to the $\sim 0.2 \% / \mathrm{dpa}$ value.

\subsection{Grain growth in severely deformed EK-181}

Fig. 11a shows typical cross-sectional TEM images obtained from severely deformed samples irradiated to 100 peak dpa at different temperatures. Fig. 11b shows typical micrographs of the severely deformed samples irradiated to doses of 100 to 400 peak dpa at $475{ }^{\circ} \mathrm{C}$. Irradiated regions are marked by the dashed boxes. Comparisons between irradiated and unirradiated regions show that there is a significant grain growth in the irradiated regions, the grain size increaseing with both increasing temperature and dpa level.

Fig. 12a compares the grain sizes of irradiated and unirradiated regions as a function of irradition temperature at the dose of 100 peak dpa. The grain size measurements were 
performed using BF TEM imaging. Statistics were obtained by performing 20 to 120 measurements on different areas to calculate the average grain size in both irradiated and unirradiated regions. Even though grains are quite equiaxed, measurements were performed along different directions and the grain size was averaged over these measurements.

The dashed lines in Fig.12a represent the range of grain sizes before irradiation. The triangular symbols refer to the grain sizes in the unirradiated region influenced by thermal annealing only. The square symbols refer to the grain sizes within the irradiated region where both irradiation and thernal annealing drive the evolution. While thermal annealing only slightly increased grain sizes, additional size growth was noticable even for the lowest irradiation temperature, and irradiation-induced growth became significant at temperatures higher than $700 \mathrm{~K}$.

Fig. $12 \mathrm{~b}$ shows grain sizes of the severely deformed samples which were irradiated to different dpa values at $475{ }^{\circ} \mathrm{C}$. Systematically, ion irradiation increased grain sizes at all dpa values. Significant irradiation-driven grain growth was observed even at the lowest dpa (100 peak dpa). Both irradiated and unirradiated regions reached saturation at high dpa values. Beyond a dose of 200 peak dpa, the average grain size in irradiated region approached $\sim 1000$ $\mathrm{nm}$ and the size in the unirradiated region approached $\sim 370 \mathrm{~nm}$.

Previous studies suggested that if the damage cascade and thermal spike volume are comparable to grain sizes, grain growth is promoted [48]. Molecular dynamics simulations also predicted such damage-driven grain growth [49]. However, the initial grain size of the severely deformed sample is about $135 \mathrm{~nm}$, which is much larger than the typical cascade volume estimated to be only a few nanometers from ion tracks.

Kiritani performed in-situ electron irradiation and TEM characterization on previously neutron-irradiated metals [50]. Based on different radiation responses under electron irradiation, the types of defect clusters (interstitial vs. vacancy) were determined. The largest defect clusters were found to be interstitial clusters of sizes up to about $10 \mathrm{~nm}$. This size is comparable to the typical size of damage cascades. Note, damage cascades created by heavy ion irradiation are expected to be smaller than that created by neutron irradiation. Hence, direct damage cascade interactions with grain boundaries are possible, but may not play a significant role in the present study, considering the relatively large grain sizes in the deformed samples. Furthermore, the damage cascade effect should be dominant mostly in the 
peak dpa region where nuclear stopping powers reach the maximum. This effect is supposed to be less dominant in the near surface region where electronic stopping powers are more significant. In contrast, TEM cross sectional characterization does not observe larger grains in the peak dpa region. Instead, Fig. 11b shows that grain growth in the shallower regions is more significant than the deeper regions, which is correlated with the observation that void swelling is more significant in the shallow regions. As to be discussed, these observations suggest a possible correlation between grain growth and void swelling.

Significant grain growth was observed to occur even for the lowest dose of 100 peak dpa. If we accept the assumption that void swelling is correlated with grain growth, two observations are expected: one is that after 100 peak dpa irradiation, the deformed sample should already exhibit noticeable swelling. The other is that the void swelling incubation period for the deformed samples may be less than 100 dpa. As shown in Fig. 7e, for the 100 peak dpa, swelling is noticeable in the shallow depth region of 100 to $400 \mathrm{~nm}$. Furthermore, the shortest swelling incubation period for the depth region of 100 to $400 \mathrm{~nm}$, as suggested by Fig. 9b, is $\sim 75$ local dpa. While these observations cannot serve as direct evidence that grain instability accelerates void swelling, they support a correlation between these two. The unanswered question is whether grain instability reduces the incubation period for void swelling or whether void swelling accelerates grain growth. Most likely there is a mutually accelerating synergism between the two processes. Void swelling is known to generate stresses in ionirradiated specimens, producing a 100\% anisotropic movement of mass toward the specimen surface by dislocation movement that probably also accelerates movement and alterations of grain boundaries $[51,52]$.

Grain coarsening reduces the benefit of introducing high density grain boundaries as defect sinks. In comparison with the effects from the changes of boundary densities, the effect from coarsening itself appears to play a significant role in swelling. Hence, even after grain coarsening of grain sizes smaller than the annealed samples, noticeable swelling occurred in deformed samples. The present studies suggest that grain boundary engineering needs to consider boundary stability. The use of oxide particles or carbide precipitates to immobilize grain boundaries may be a realistic approach to retain the benefits of severe plastic deformation to reduce void swelling. Indeed, other studies have shown the validity of this approach to reduce void swelling in nano-grained alloys [53, 54]

\section{Conclusion}


Self-ion irradiations of two variants of EK-181 alloy have been examined with special interest paid to the neutron-atypical factors of injected interstitial and internal gradients in dpa rate. While severe plastic deformation changes the depth distribution of swelling in EK181 , it does not confer any significant improvement in swelling resistance, and appears to actually accelerate the onset of swelling in the shallow depth region. This result is attributed to the instability of nano-grains in the severely deformed samples arising from the combined effects of thermal annealing and ion irradiation. It appears that once steady-state swelling is attained in any alloy variant or depth position in that variant, the swelling rate is on the order of $\sim 0.2 \%$ per dpa. However, the incubation period preceding this rate is dependent on the dpa rate associated with the depth range where the data were extracted.

\section{Acknowledgements}

The Texas A\&M University team was supported by US Department of Energy, NEUP program, through grant DE-NE0008297. Valiev and Enikeev acknowledge the partial support through contract number 14.B25.31.0017 by Russian Ministry for Education and Science. 


\section{References:}

[1] S.V. Rogozhkin, V.S. Ageev, A.A. Aleev, A.G. Zaluzhnyi, M.V. Leont'eva-Smirnova, A.A. Nikitin, S.V., Phys. Met. Metallogr. 108 (6) (2009) 579-585.

[2] A. Kohyama, A. Hishinuma, Y. Kohno, K. Shiba, A. Sagara, Sci. Rep. RITU 1 (1997) $137-141$.

[3] R.L. Klueh, D.L. Harries, High Chromium Ferritic and Martensitic Steels for Nuclear Applications. West Conshohocken, PA, ASTM, 2001.

[4] A.G. Ioltukhovskiy, A.I. Blokhin, N.I. Budylkin, V.M. Chernov, M.V. Leont'evaSmirnova, E.G. Mironova, E.A. Medvedeva, M.I. Solonin, S.I. Porollo, L.P. Zavyalsky, J. Nucl. Mater. 283-287 (2000) 652-656.

[5] C. Petersen, in: Proceedings of IEA Working Group Meeting on RAF/M Steels, JAERI, Tokyo (1997) 134.

[6] A.V. Vatulin, Vopr. At. Nauki Tekh., Ser. Materialoved. Nov. Mater., 1 (62) (2004) 2641.

[7] V.V. Sumin, V.G. Simkin, S.G. Sheverev, M.V. Leont'eva-Smirnova, V.M. Chernov, Phys. Met. Metal., 108 (6), (2009) 600-605.

[8] B.K. Kardashev, V.M. Chernov, Mat. Sci. Eng. A 521-522 (2009) 329-334.

[9] V.M. Chernov, M.V. Leont'eva-Smirnova, M.M. Potapenko, N.I. Budylkin, Yu.N. Devyatko, et al., Nucl. Fusion 47 (2007) 839-848.

[10] N.V. Boiko, Yu.N. Devyatko, I.A. Evstyukhina, O.N. Izmaylov, S.G. Rudakov, M.N. Smirnov, Inorg. Mater.: Appl. Res. 3 (2) (2012) 102-106.

[11] V.M. Chernov, G.N. Ermolaev, M.V. Leont'eva-Smirnova, Tech. Phys. 55 (7) (2010) 985-990.

[12] A.V. Golubeva, N.P. Bobyr, D.I. Cherkez, A.V. Spitsyn, M. Mayer, Yu.M. Gasparyan, V.S. Efimov, V.M. Chernov, M.V. Leont'eva-Smirnova, J. Nucl. Mater. 438 (2013) S983-S987.

[13] A.V. Panin, V.A. Romanova, R.R. Balokhonov, O.B. Perevalova, E.A. Sinyakova, O.S. Emelyanova, M.V. Leont'eva-Smirnova, N.I. Karpenko, Phys. Mesomech., 15 (1-2) (2012) 94-103.

[14] A.V. Panin, V.M. Chernov, M.V. Leont'eva-Smirnova, E.A. Melnikova, J. Nucl. Mater. 386-388 (2009) 466-470.

[15] O.V. Emelyanova, P.S. Dzhumaev, V.L. Yakushin, B.A. Kalin, M.G. Ganchenkova, A.T. Khein, M.V. Leont'eva-Smirnova, R.Z. Valiev, N.A. Enikeev, L. Shao, E. Aydogan, M. Short, F.A. Garner, Nucl. Instrum. Meth. B 365 (2015) 218-221. 
[16] M. Rose, A.G. Balogh, H. Hahn, Mater. Sci. Forum 248-249 (1997) 213-216.

[17] A.R. Kilmametov, D.V. Gunderov, R.Z. Valiev, A.G. Balogh, H. Hahn, Scr. Mat. 59 (2008) 1027-1030.

[18] B. Radiguet, A. Etienne, P. Pareige, X. Sauvage, R. Valiev, J. Mater. Sci. 43(23-24) (2008) 7338-7343.

[19] P.B. Revathy Rajan, I. Monnet, E. Hug, A. Etienne, N. Enikeev, C. Keller, X. Sauvage, R.Z. Valiev, B. Radiguet, IOP Conf. Ser.: Mater. Sci. Eng. 63 (2014) 012121.

[20] N. Nita, R. Schaeublin, M. Victoria, J. Nucl. Mater. 329-333 (2004) 953-957.

[21] R.Z. Valiev, M.J. Zehetbauer, Y. Estrin, H.W. Hoppel, Y. Ivanisenko, H. Hahn, G. Wilde, H.J. Roven, X. Sauvage, T.G. Langdon, Adv. Eng. Mater. 9 (2007) 527-533.

[22] Y.T. Zhu, T.C. Lowe, T.G. Langdon, Scr. Mat. 51 (2004) 825-830.

[23] C. Sun, K.Y. Yu, J.H. Lee, Y. Liu, H. Wang, L. Shao, S.A. Maloy, K.T. Hartwig, X. Zhang, J. Nucl. Mater. 420 (2012) 235-240.

[24] C. Sun, Y. Yang, Y. Liu, K.T. Hartwig, H. Wang, S.A. Maloy, T.R. Allen, X. Zhang, Mater. Sci. Eng. A 542 (2012) 64-70.

[25] D.C. Foley, K.T. Hartwig, S.A. Maloy, P. Hosemann, X. Zhang, J. Nucl. Mater. 389 (2009) 221-224.

[26] M. Song, R. Zhu, D.C. Foley, C. Sun, Y. Chen, K.T. Hartwig, X. Zhang, J. Mater. Sci. 48 (2013) 7360-7373.

[27] M. Song, C. Sun, J. Jang, C.H. Han, T.K. Kim, K.T. Hartwig, X. Zhang, J. Alloys Compd. 577 (2013) 247-256.

[28] S.V. Rogozhkin, T.V. Kulevoy, N.A. Iskandarov, N.N. Orlov, B.B. Chalykh, A.A. Aleev, N. Yu. Grachev, R.P. Kujbeda, A.A. Nikitin, A.D. Fertman, V.B. Shishmarev, At. Energ. 114 (1) (2013) 14-20.

[29] A. Alsabbagh, R.Z. Valiev, K.L Murty, J. Nucl. Mater. 443 (2013) 302-310.

[30] A. Alsabbagh, A. Sarkar, B. Miller, J. Burns, L. Squires, D. Porter, J.I. Cole, K.L. Murty, Mater. Sci. Eng. A 615 (2014) 128-138.

[31] V.K. Shamardin, Y.D. Goncharenko, T.M. Bulanova, A.A. Karsakov, I.V. Alexandrov, M.M. Abramova, M.V. Karavaeva, Rev. Adv. Mater. Sci. 31 (2012) 167-173.

[32] R.Z. Valiev, R.K. Islamgaliev, I.V. Alexandrov, Progr. Mater. Sci 45 (2000) 103-189.

[33] J.F. Ziegler, J.P. Biersack, "SRIM2013: The Stopping and Range of Ions in Matter: Version 2013", available at http://www.srim.org. 
[34] L.R. Greenwood, R.K. Smither, SPECTER: Neutron Damage Calculations for Materials Irradiations, ANL/FPP/TM-197, Argonne National Laboratory, Argonne, IL, January 1985, available at http://www.osti.gov.

[35] R.E. Stoller, M.B. Toloczko, G.S. Was, A.G. Certain, S. Dwaraknath, F.A. Garner, Nucl. Instrum. Meth. B, 310 (2013) 75-80.

[36] J.A. Sprague, F.A. Smidt, Jr., Naval Research Laboratory Semi-Annual Progress Report, NRL Memorandum Report 2629 (November 1972 - April 1973) 27.

[37] ASTM E521-83. Standard Practice for Neutron Radiation Damage Simulation by Charged-Particle Irradiation. West Conshohocken, PA, ASTM International.

[38] E. Getto, Z. Jiao, A.M. Monterrosa, K. Sun, G.S. Was, J. Nucl. Mater. 465 (2015) 116126.

[39] J.G. Gigax, E. Aydogan, T. Chen, D. Chen, L. Shao, Y. Wu, W.Y. Lo, Y. Yang, F.A. Garner, J. Nucl. Mater. 465 (2015) 343-348.

[40] L. Shao, C-C Wei, J. Gigax, A. Aitkaliyeva, D. Chen, B.H. Sencer, F. A Garner, J. Nucl. Mater. 453 (2014) 176-181.

[41] F.A. Smidt, P.R. Malmberg, J.A. Sprague, J.E. Westmoreland in: Irradiation Effects on the Microstructure and Properties of Metals ASTM STP-611, American Society for Testing and Materials, Philadelphia, (1976) 227-241.

[42] V. Bryk, O. Borodin, A. Kalchenko, V. Voyevodin, V. Ageev, A. Nikitina, V. Novikov, V. Inozemtsev, A. Zeman, F. Garner in: Proceedings of Accelerator Applications, Bruges, Belgium, (2013) 1-6.

[43] F. A. Garner, J. Nucl. Mater. 117 (1983) 177-197.

[44] F. A. Garner, G. L. Guthrie, in Radiation Effects and Tritium Technology for Fusion Reactors, CONF-750989, (1976) I-491-518.

[45] F.A. Garner, "Radiation Damage in Austenitic Steels", in Konings, R.J.M., (ed.) Comprehensive Nuclear Materials, Volume 4, (2012) 33-95.

[46] F.A. Garner, M.B. Toloczko, B.H. Sencer, J. Nucl. Mater. 276 (2000) 123-142.

[47] F. A. Garner et al., "Summary of Alloy Development Intercorrelation Experiment", in Proceedings of Workshop on Correlation of Neutron and Charged Particle Damage, Oak Ridge, TN, (1976) 147-175.

[48] D. Kaoumi, A.T. Motta, R.C. Birtcher, J. Appl. Phys. 104 (2008) 073525 (1-13).

[49] W. Voegeli, K. Albe, H. Hahn, Nucl. Instrum. Meth. B 202 (2003) 230-235.

[50] M. Kiritani, J. Nucl. Mat. 251 (1997) 237-251. 
[51] F. A. Garner, G. L. Wire and E. R. Gilbert, Radiation Effects and Tritium Technology for Fusion Reactors, CONF-750989, 1976, p. I-474.

[52] W. G. Wolfer and F. A. Garner, J. Nucl. Mater., $85-86$ (1979) 583-589.

[53] M. B. Toloczko, F. A. Garner, V. N. Voyevodin, V. V. Bryk, O. V. Borodin, V. V. Mel'nychenko, and A. S. Kalchenko, J. Nucl. Mater. 453 (2014) 323-333.

[54] F. A. Garner, L. Shao, M. B. Toloczko, S. A. Maloy, V. N. Voyevodin, "Use of self-ion bombardment to study void swelling in advanced radiation-resistant alloys", Proc. 17th International Conference on Environmental Degradation of Materials in Nuclear Power Systems - Water Reactors, August 9-12, 2015, Ottawa, Ontario, Canada. 
Table 1: Composition of EK-181 samples (wt\%)

\begin{tabular}{|c|c|c|c|c|c|c|c|c|c|c|c|c|}
\hline $\mathbf{C}$ & $\mathbf{C r}$ & $\mathbf{N i}$ & $\mathbf{M o}$ & $\mathbf{N b}$ & $\mathbf{W}$ & $\mathbf{V}$ & $\mathbf{T a}$ & $\mathbf{B}$ & $\mathbf{S i}$ & $\mathbf{N}$ & $\mathbf{M n}$ & $\mathbf{Z r}$ \\
\hline 0.14 & 11.2 & 0.03 & 0.04 & 0.01 & 1.17 & 0.29 & 0.17 & 0.004 & 0.37 & 0.044 & 0.94 & 0.05 \\
\hline
\end{tabular}




\section{Captions:}

Table 1. Composition of EK-181 samples (wt\%).

Fig. 1. Schematic view of the high pressure torsion process used to produce severely plastically deformed samples of EK-181.

Fig. 2. SRIM-calculated depth profiles of dpa and implanted atoms in pure Fe irradiated with 3.5 $\mathrm{MeV} \mathrm{Fe}^{2+}$ ions for 100 peak dpa.

Fig. 3. SEM images of (a) annealed EK-181 (b) HPT processed, severely deformed EK-181, showing the non-homogenous distribution of carbides along the grain boundaries on both variants. Typical $\mathrm{M}_{23} \mathrm{C}_{6}$ type carbides along the grain boundaries are shown in bright contrast by arrows. Note difference in scale of the two micrographs.

Fig. 4. TEM micrographs of (a) annealed EK-181 samples after irradiation up to 100 peak dpa at $400,425,450,475$ and $500{ }^{\circ} \mathrm{C}$, and the corresponding swelling vs. temperature plot; and (b) severely deformed EK-181 samples after irradiation up to 100 peak dpa at 400, 425, 450,475 and $500{ }^{\circ} \mathrm{C}$, and the corresponding swelling vs. temperature plot. Arrows illustrate the beam direction.

Fig. 5. TEM images of annealed EK-181 samples irradiated to (a) 100 peak dpa, (b) 200 peak dpa, (c) 300 peak dpa and (d) 400 peak dpa at $475{ }^{\circ} \mathrm{C}$; and (e) corresponding swelling vs. depth profiles. Arrows illustrate the beam direction.

Fig. 6. Plots of (a) void size and (b) void density as a function of local dpa for annealed EK181. Measurements were performed at the depth region between 400 to $700 \mathrm{~nm}$.

Fig. 7. TEM micrographs of severely deformed EK-181 after irradiation to (a) 100 peak dpa, (b) 200 peak dpa, (c) 300 peak dpa and (d) 400 peak dpa at $475^{\circ} \mathrm{C}$; and (e) corresponding swelling vs. depth profiles. Arrows illustrate the beam direction.

Fig. 8. Plots of (a) void size and (b) void density as a function of local dpa for severely deformed EK-181. Measurements were performed at a depth region between 400 to $700 \mathrm{~nm}$.

Fig. 9. Swelling curves of (a) annealed and (b) severely deformed EK-181 samples as a function of dpa, extracted from different depth regions.

Fig. 10. (a) Depth dependent swelling of annealed specimen after 400 peak dpa irradiation and (b) swelling of the same sample as a function of its local dpa values. Open circles denote 
data from the depth range where injected interstitial suppression is strong. Solid circles denote swelling at depths where such suppression is not significant.

Fig. 11. TEM micrographs showing extensive grain growth in the irradiated regions (a) for 100 peak dpa irradiation at different temperatures, and (b) for different dose levels at $475{ }^{\circ} \mathrm{C}$. Arrows illustrate the beam direction.

Fig. 12. (a) Temperature dependence of mean grain size at 100 peak dpa, and (b) dose dependence of mean grain size at $475{ }^{\circ} \mathrm{C}$ in both irradiated and unirradiated regions of severely deformed EK-181 samples. Dotted lines bound the range of pre-irradiation sizes. 


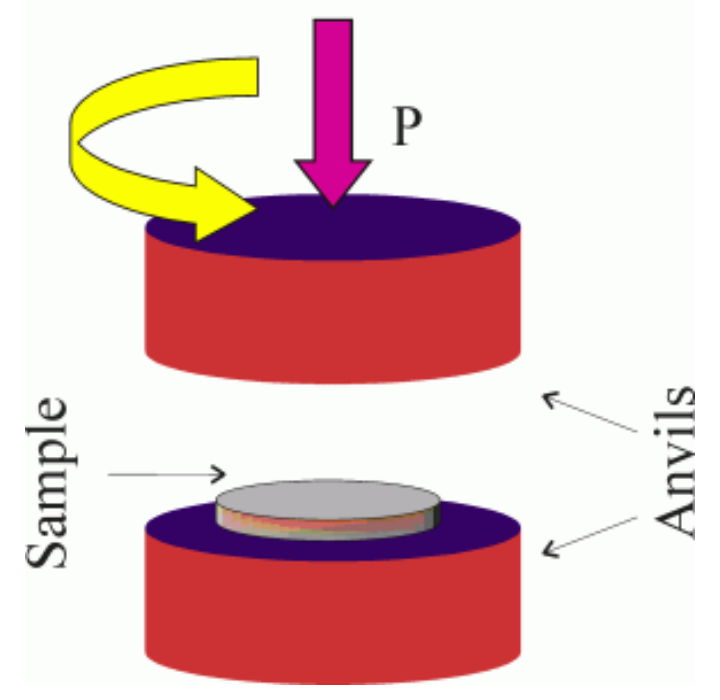

Fig. 1. Schematic view of the high pressure torsion process used to produce severely plastically deformed samples of EK-181. 


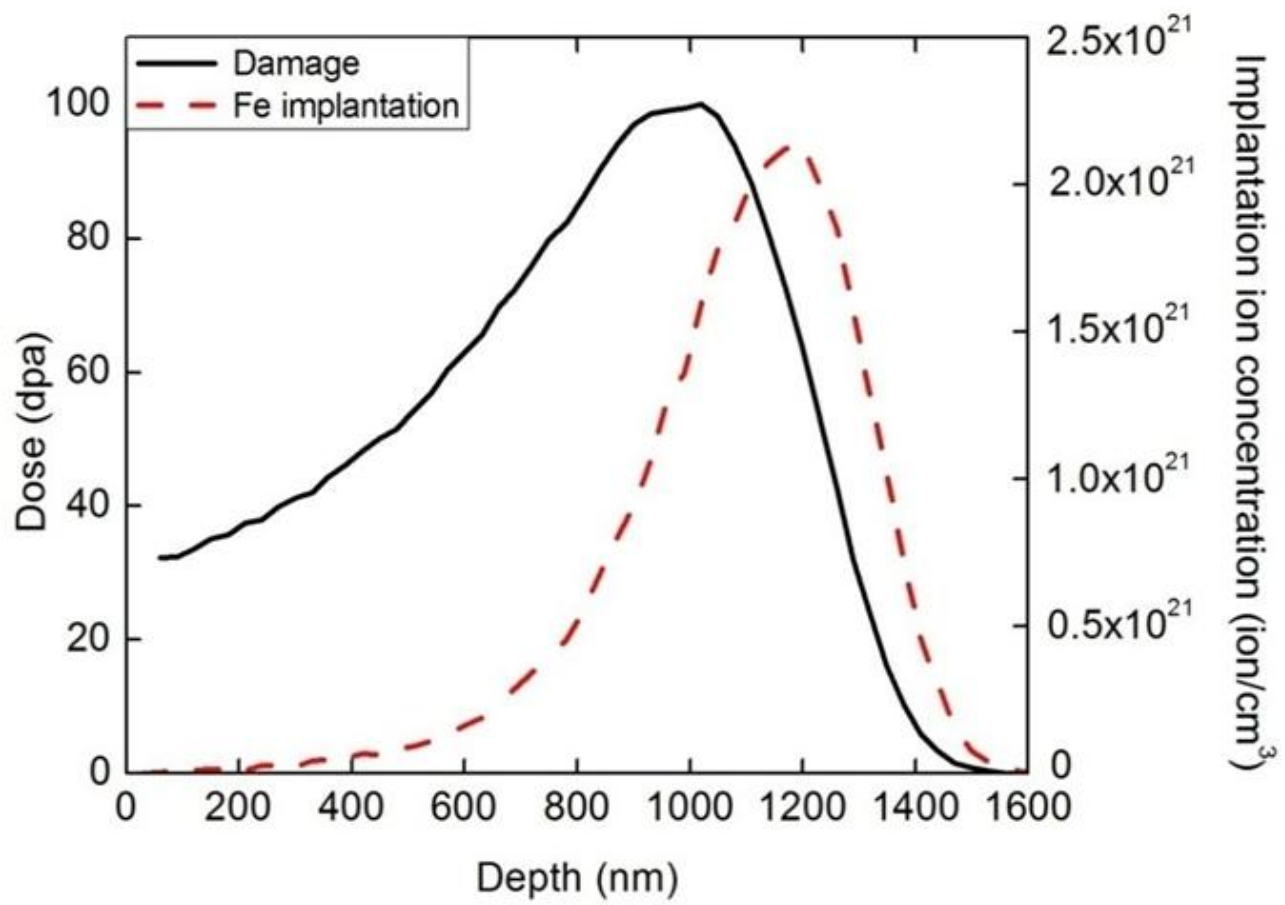

Fig. 2. SRIM-calculated depth profiles of dpa and implanted atoms in pure Fe irradiated with 3.5 $\mathrm{MeV} \mathrm{Fe}^{2+}$ ions for 100 peak dpa. 

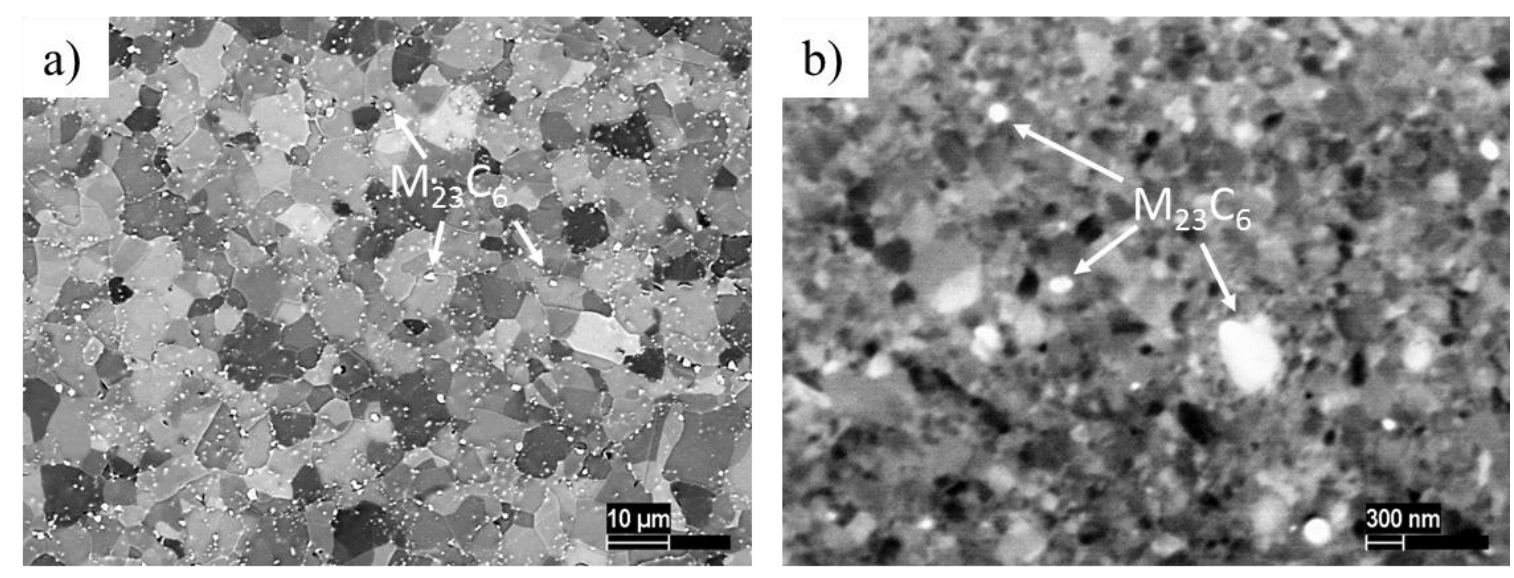

Fig. 3. SEM images of (a) annealed EK-181 (b) HPT processed, severely deformed EK-181, showing the non-homogenous distribution of carbides along the grain boundaries on both variants. Typical $\mathrm{M}_{23} \mathrm{C}_{6}$ type carbides along the grain boundaries are shown in bright contrast by arrows. Note difference in scale of the two micrographs. 
a)
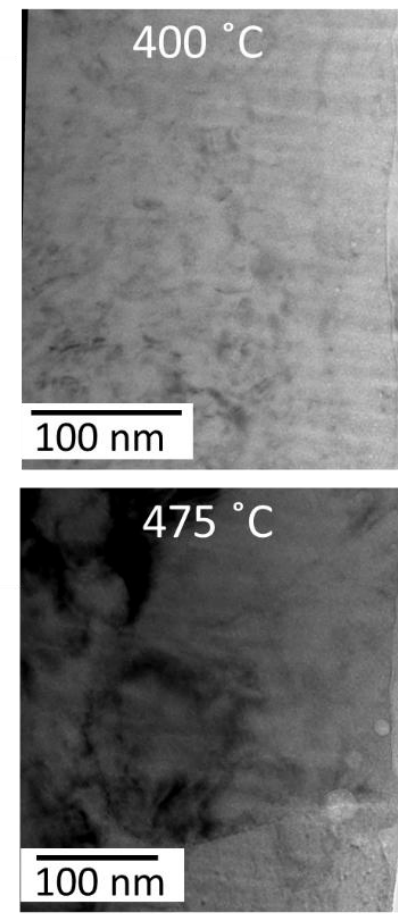

b)
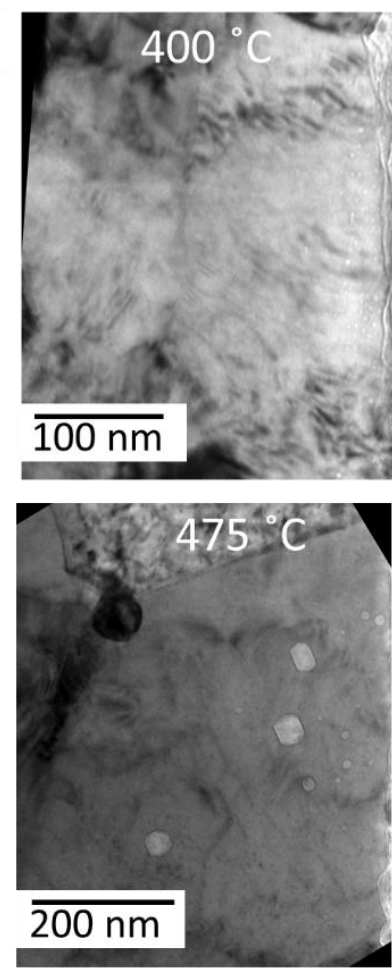
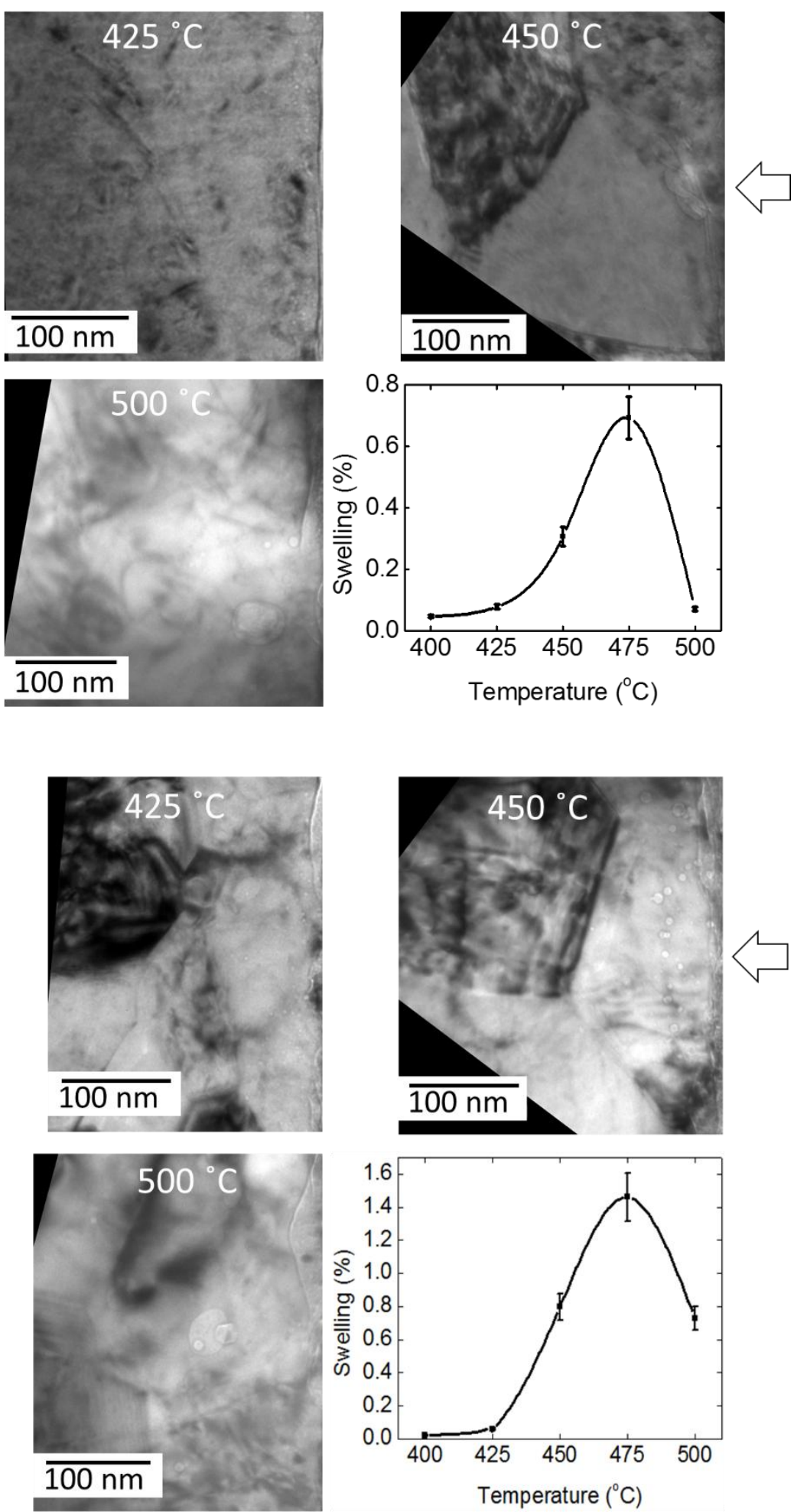

Fig. 4. TEM micrographs of (a) annealed EK-181 samples after irradiation up to 100 peak dpa at $400,425,450,475$ and $500{ }^{\circ} \mathrm{C}$, and the corresponding swelling vs. temperature plot; and (b) severely deformed EK-181 samples after irradiation up to 100 peak dpa at 400, 425, 450,475 and $500{ }^{\circ} \mathrm{C}$, and the corresponding swelling vs. temperature plot. Arrows illustrate the beam direction. 

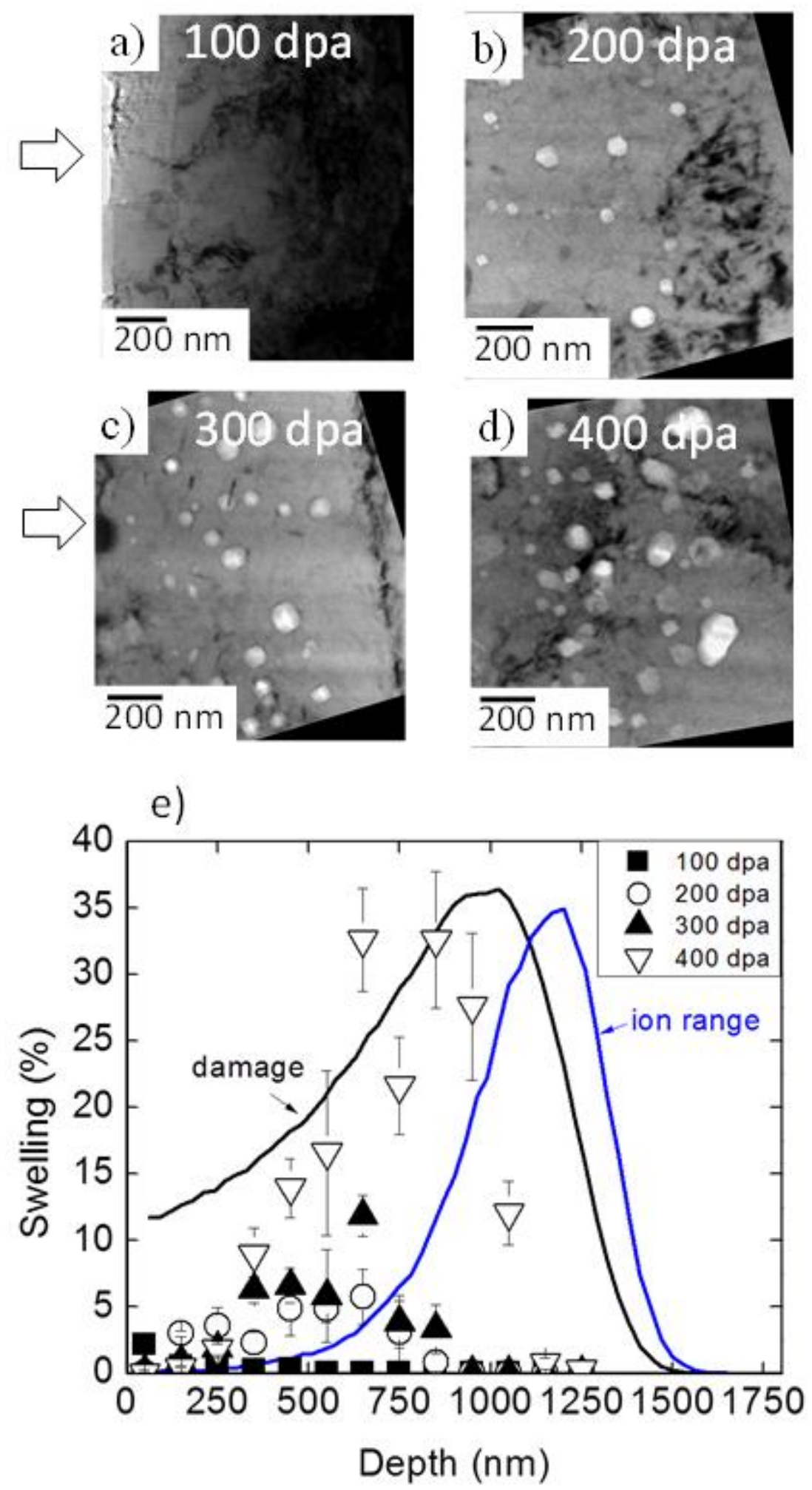

Fig. 5. TEM images of annealed EK-181 samples irradiated to (a) 100 peak dpa, (b) 200 peak dpa, (c) 300 peak dpa and (d) 400 peak dpa at $475^{\circ} \mathrm{C}$; and (e) corresponding swelling vs. depth profiles. Arrows illustrate the beam direction. 

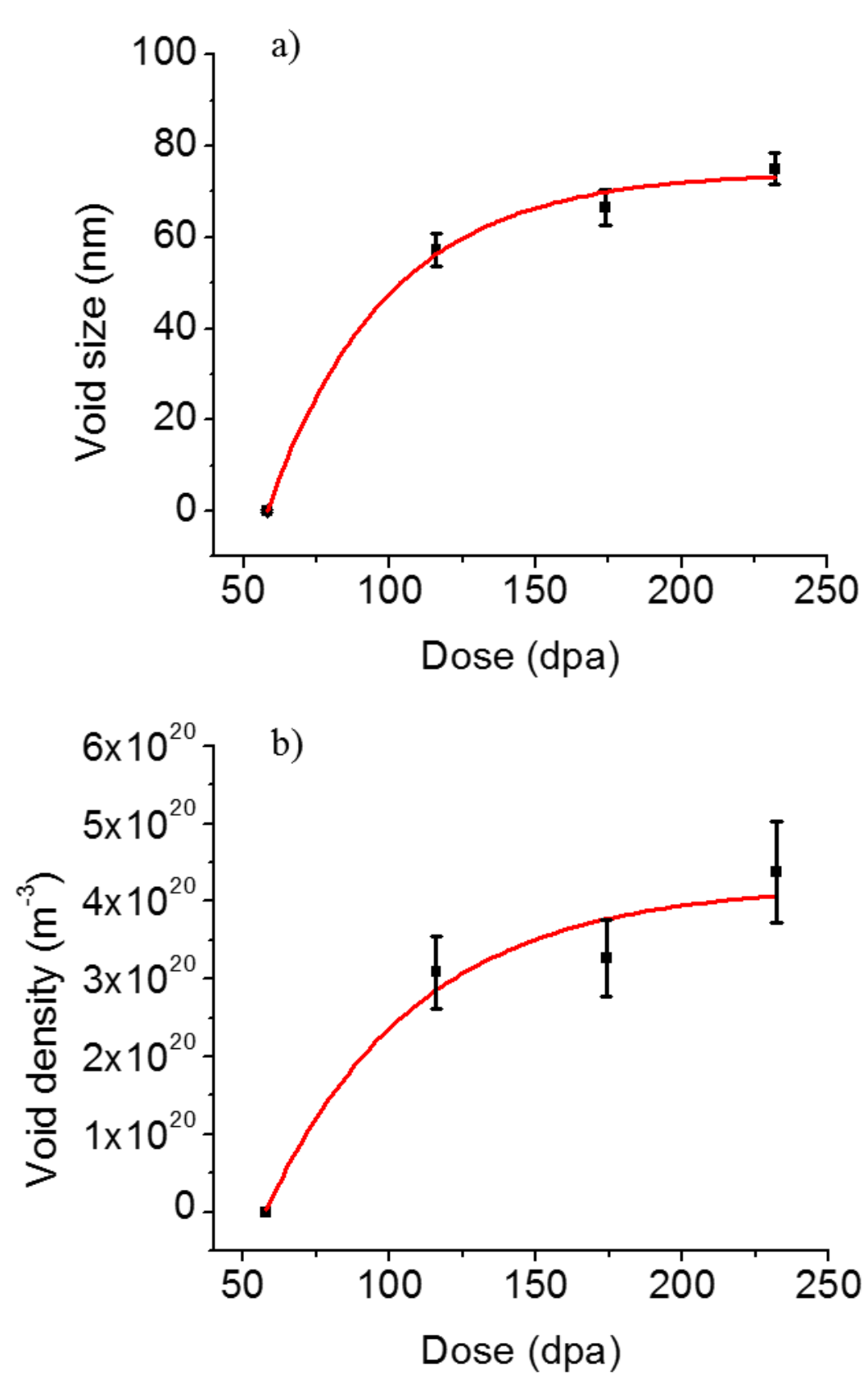

Fig. 6. Plots of (a) void size and (b) void density as a function of local dpa for annealed EK181. Measurements were performed at the depth region between 400 to $700 \mathrm{~nm}$. 

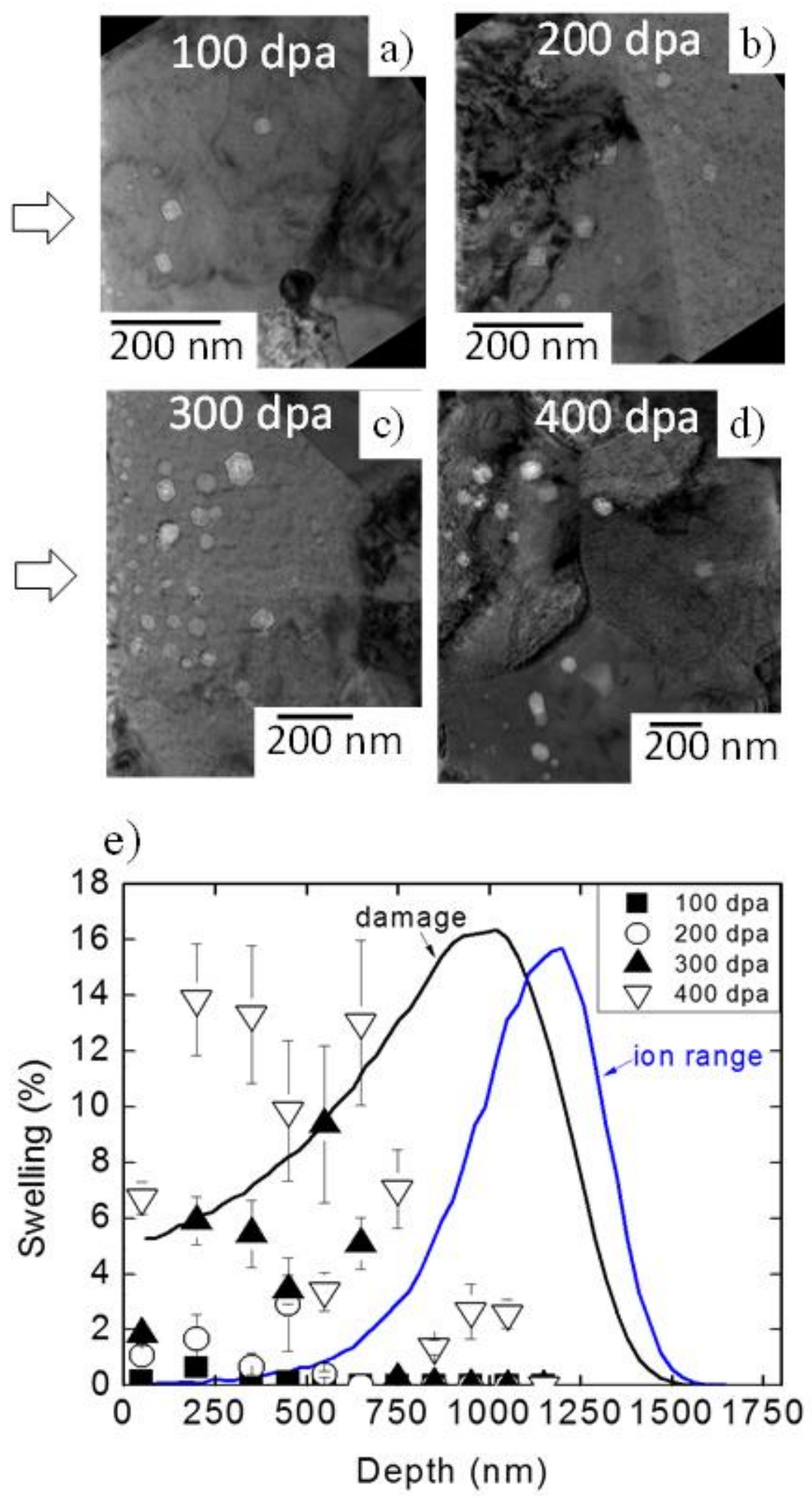

Fig. 7. TEM micrographs of severely deformed EK-181 after irradiation to (a) 100 peak dpa, (b) 200 peak dpa, (c) 300 peak dpa and (d) 400 peak dpa at $475^{\circ} \mathrm{C}$; and (e) corresponding swelling vs. depth profiles. Arrows illustrate the beam direction. 

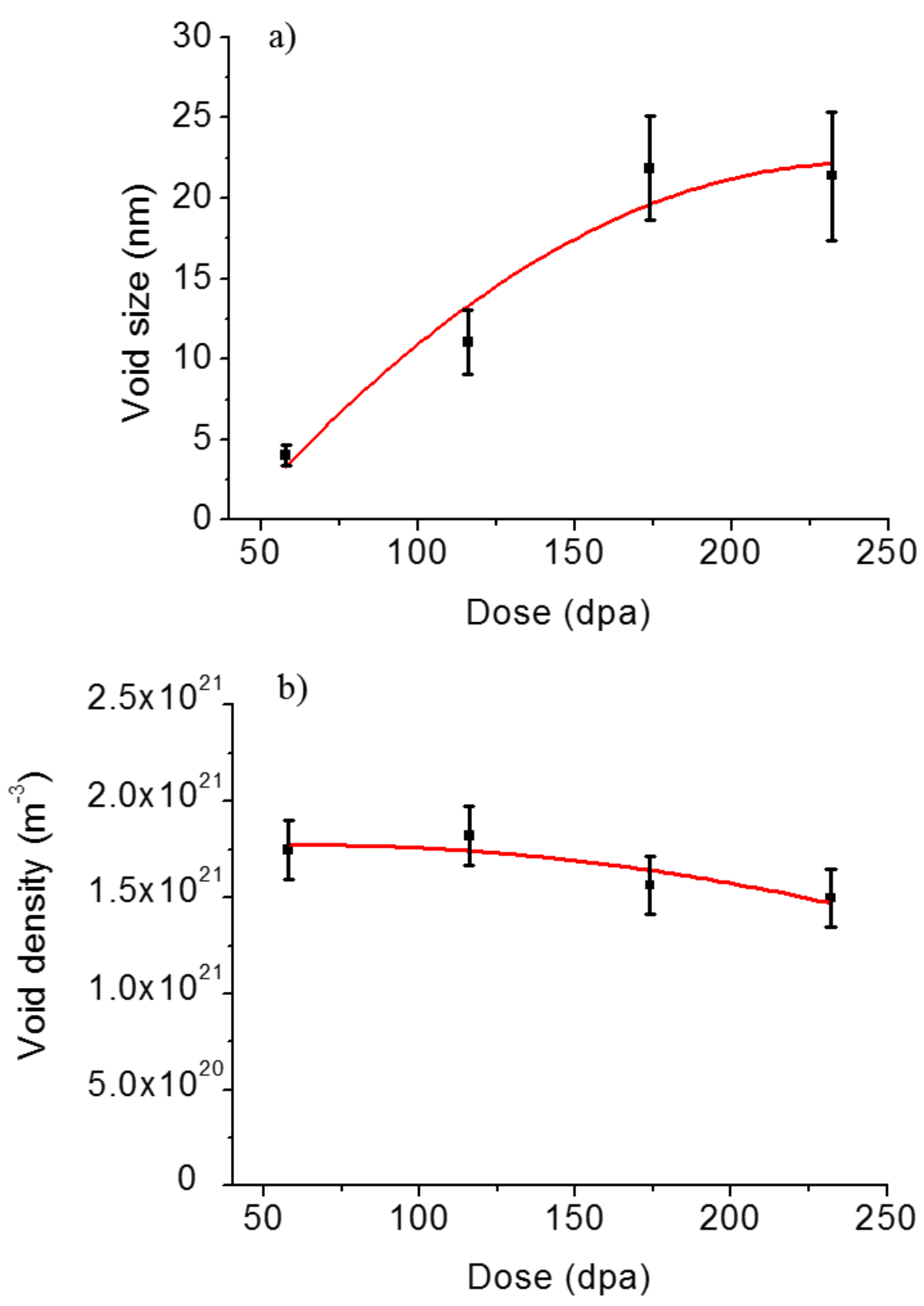

Fig. 8. Plots of (a) void size and (b) void density as a function of local dpa for severely deformed EK-181. Measurements were performed at a depth region between 400 to $700 \mathrm{~nm}$. 


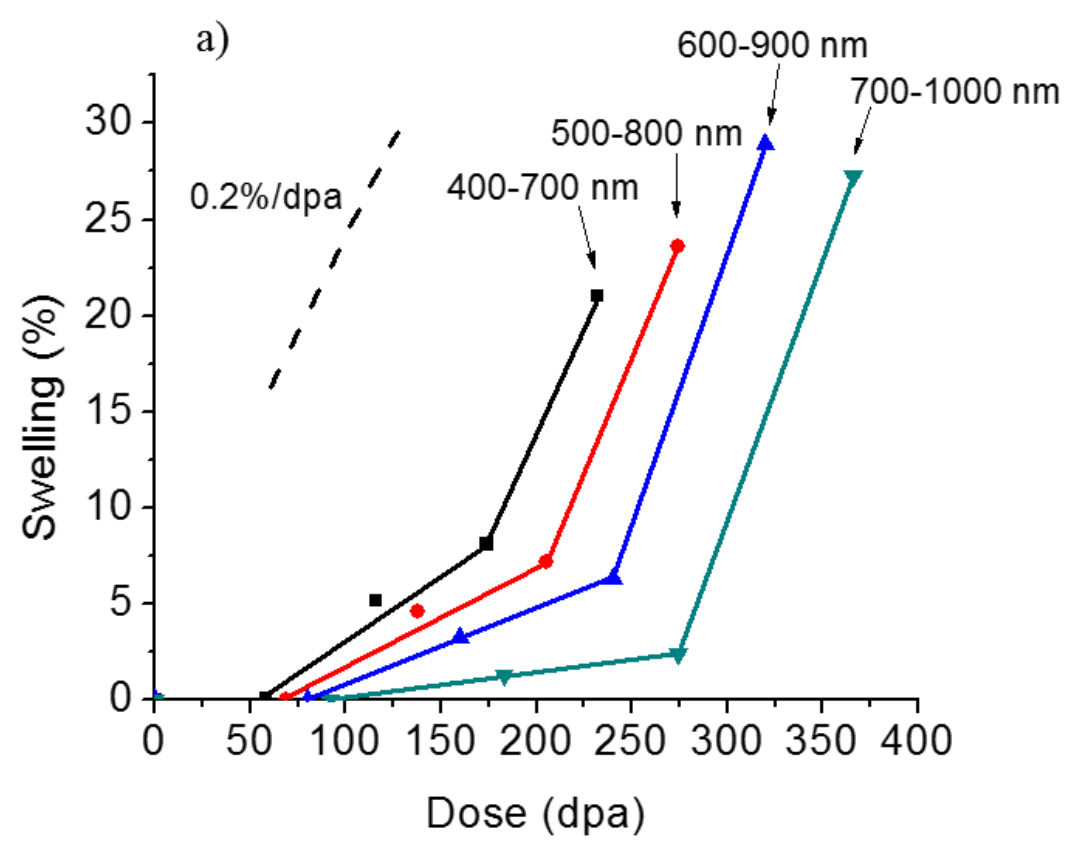

b)

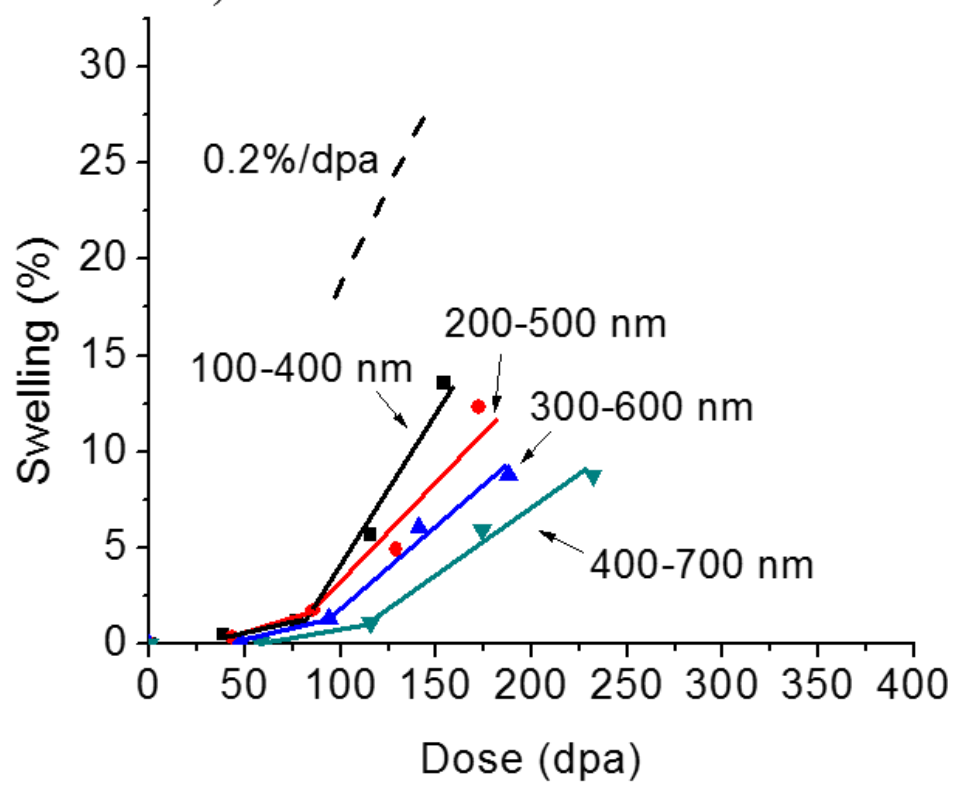

Fig. 9. Swelling curves of (a) annealed and (b) severely deformed EK-181 samples as a function of dpa, extracted from different depth regions. 

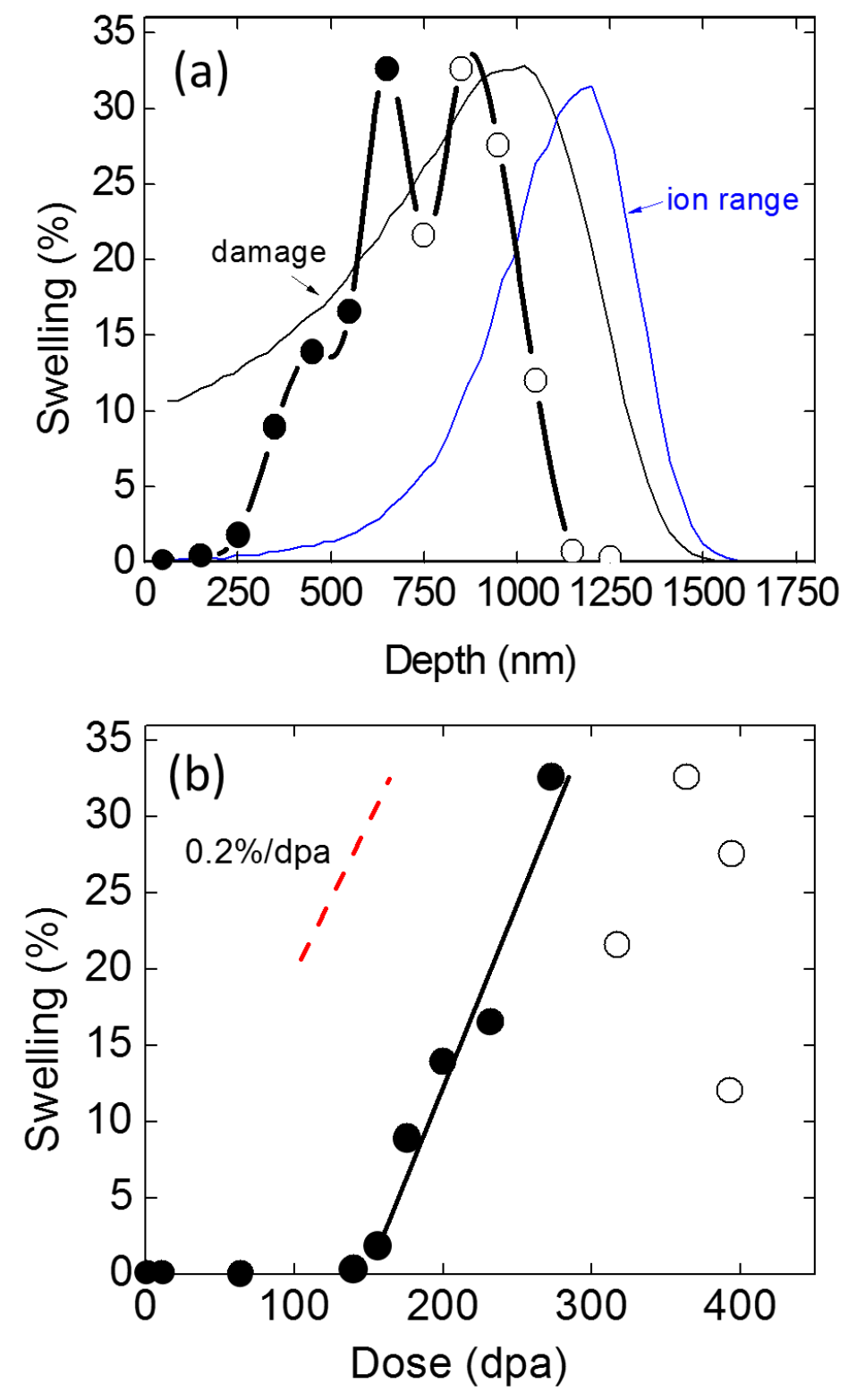

Fig. 10. (a) Depth dependent swelling of annealed specimen after 400 peak dpa irradiation and (b) swelling of the same sample as a function of its local dpa values. Open circles denote data from the depth range where injected interstitial suppression is strong. Solid circles denote swelling at depths where such suppression is not significant. 


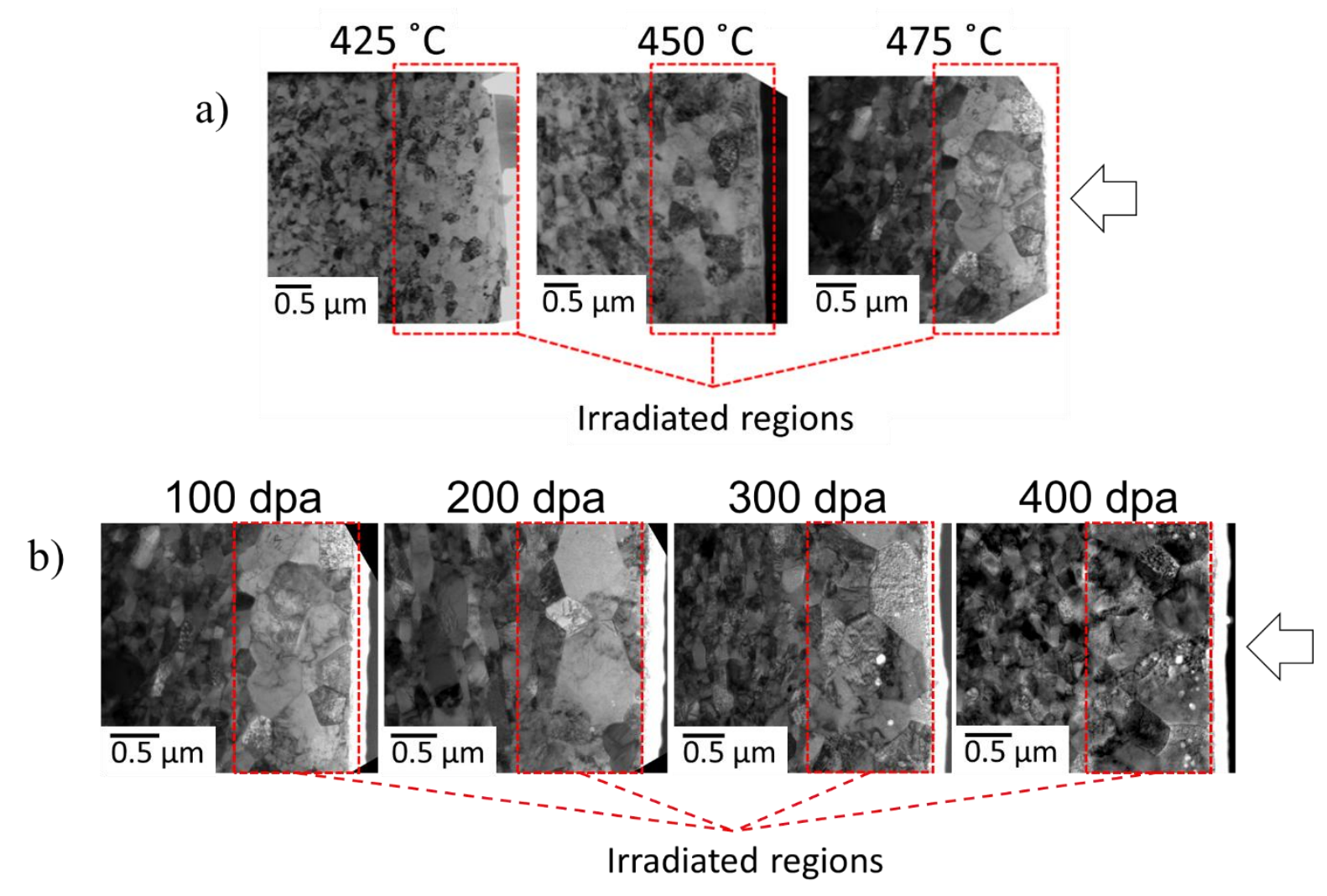

Fig. 11. TEM micrographs showing extensive grain growth in the irradiated regions (a) for 100 peak dpa irradiation at different temperatures, and (b) for different dose levels at $475{ }^{\circ} \mathrm{C}$. Arrows illustrate the beam direction. 

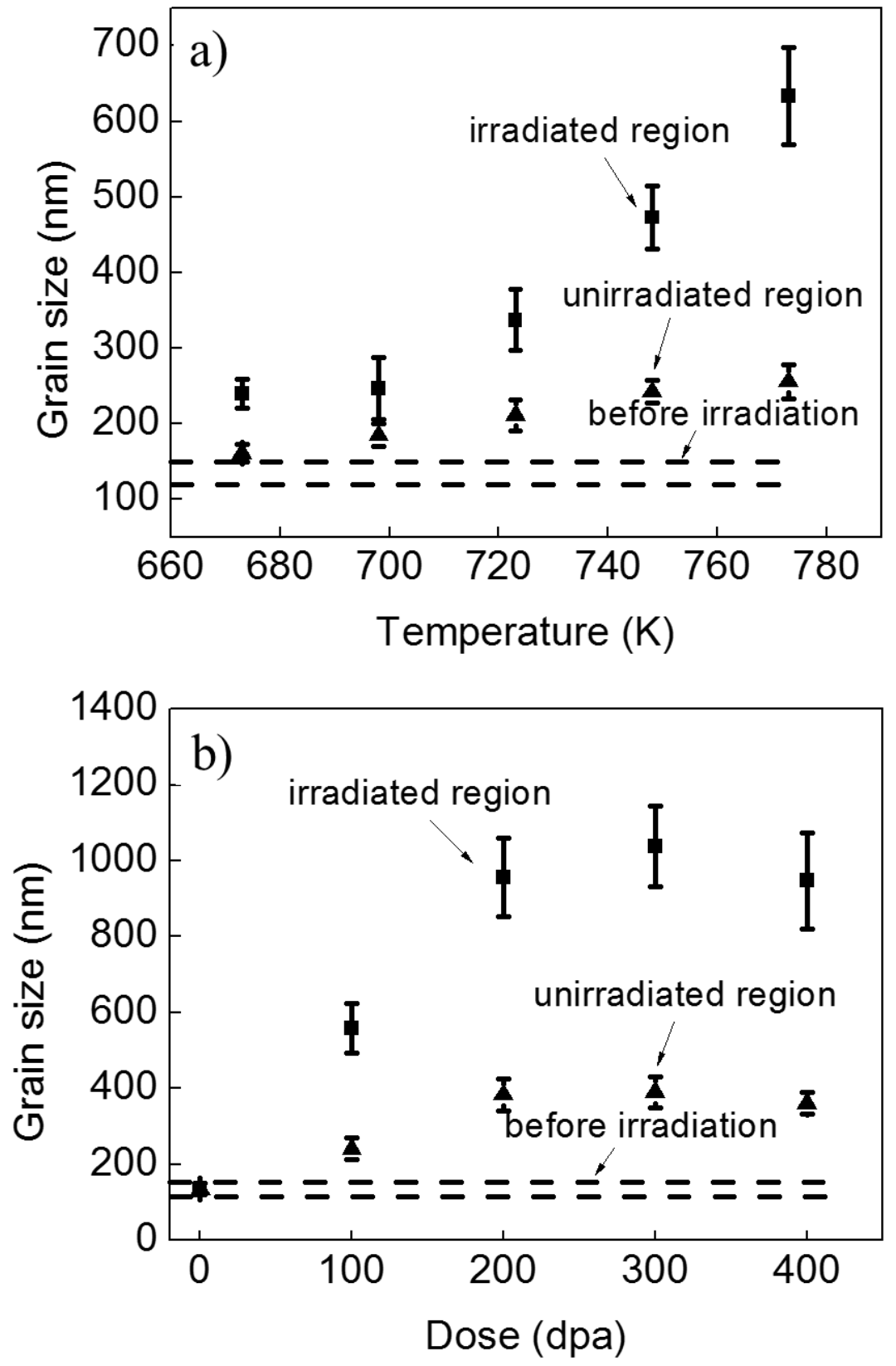

Fig. 12. (a) Temperature dependence of mean grain size at 100 peak dpa, and (b) dose dependence of mean grain size at $475{ }^{\circ} \mathrm{C}$ in both irradiated and unirradiated regions of severely deformed EK-181 samples. Dotted lines bound the range of pre-irradiation sizes. 